\title{
Резкое уменьшение подвижности дырок при снижении внешним напряжением их двумерной концентрации в дельта-допированных бором слоях алмаза
}

\author{
(C) B.A. Кукушкин \\ Институт прикладной физики Российской академии наук, \\ 603950 Нижний Новгород, Россия \\ Нижегородский государственный университет им. Н.И. Лобачевского, \\ 603950 Нижний Новгород, Россия \\ E-mail: vakuk@appl.sci-nnov.ru
}

Поступила в Редакцию 9 июля 2018 г. В окончательной редакции 6 февраля 2019 г. Принята к публикации 25 апреля 2019 г.

\begin{abstract}
Аналитически и численно показано, что подвижность дырок в дельта-допированных (т.е. имеющих малую толщину порядка нескольких постоянных кристаллической решетки) бором слоях алмаза падает с уменьшением их двумерной концентрации в процессе обеднения дельта-допированного слоя внешним напряжением. Данное падение наиболее резко выражено для максимальных начальных двумерных концентраций дырок $\sim 3 \cdot 10^{13} \mathrm{~cm}^{-2}$ (ограниченных сверху условием возможности их существенного уменьшения без электрического пробоя алмаза) и объясняется снижением степени экранирования рассеивающих кулоновских потенциалов ионизованных атомов бора и увеличением эффективности рассеяния на них вырожденных дырок вследствие уменьшения кинетической энергии последних. Соответствующие вычисления транспортного сечения рассеяния дырок проведены без использования борновского приближения (т. е. теории возмущений), условия применимости которого в дельта-допированных бором слоях алмаза не выполняются. Предсказанный эффект может быть использован при конструировании полевых транзисторов с дельта-допированными проводящими каналами на алмазе для увеличения эффективности модуляции тока исток-сток напряжением на управляющем затворе.
\end{abstract}

Ключевые слова: двумерное экранирование кулоновского потенциала, дельт-допированный слой, осажденный из газовой фазы алмаза, подвижность дырок, полевой транзистор.

DOI: 10.21883/FTP.2019.10.48304.8954

\section{1. Введение}

Как хорошо известно [1], алмаз обладает уникальными свойствами, среди которых очень высокие электростатическое пробойное поле и теплопроводность, химическая и радиационная стойкость и др. Это делает его перспективным материалом для изготовления оптических и электронных устройств с рекордными характеристиками. Среди последних особенно важны транзисторы основа для построения электронных схем.

В последние годы на алмазе, осажденном из газовой фазы на выращенную при высоких давлении и температуре алмазную подложку, были созданы биполярные [2] и полевые [3,4] транзисторы. Для повышения быстродействия последних (лимитируемого временем прохода носителями заряда подзатворной области и потому увеличивающегося с ростом их подвижности) было предложено использовать $\delta$-допирование проводящего канала, т. е. создание в нем тонкого допированного слоя с толщиной порядка нескольких постоянных кристаллической решетки. Такие слои хорошо известны в физике обычных полупроводников [5] и приводят к увеличению подвижности носителей заряда по сравнению с однородным допированием с той же концентрацией допирующих атомов, что и в центральной плоскости $\delta$-допированного слоя. Причина этого заключается в квантовом эффекте частичного проникновения волновых функций размерно квантованных состояний носителей заряда за пределы этого слоя, представляющего для них одномерную потенциальную яму. В результате носители заряда частично движутся в окружающем $\delta$-допированный слой недопированном материале и поэтому их рассеяние на находящихся в этом слое ионизованных атомах допирующей примеси уменьшается, а подвижность возрастает.

В осажденном из газовой фазы алмазе $\delta$-допированные бором (акцептором в алмазе) слои были созданы многими исследовательскими группами [6-13] и в дальнейшем использованы в проводящих каналах полевых транзисторов $[14,15]$. То, что описанный выше механизм формирования частичного пространственного разделения ионизованных атомов бора и дырок при $\delta$-допировании в алмазе действительно имеет место, подтверждается работой [13], в частности рис. $3, b$ из нее. На нем приведено распределение концентрации атомов бора в одном из $\delta$-допированных слоев алмаза в поперечном к его плоскости направлении, полученное с помощью вторичной ионной масс-спектрометрии, и распределение концентрации дырок в этом же направлении, установленное путем вольт-фарадных измерений. Из него 
видно, что ширина (по половине от максимального значения) распределения концентрации дырок $\sim 5 \mathrm{Hм}$, а аналогичная величина для атомов бора $\sim 3 \mathrm{Hм}$, т.е. дырки распределены почти в 2 раза более широко, чем атомы бора.

Подвижность дырки в $\delta$-допированных слоях определяется в основном ее рассеянием на кулоновских потенциалах ионизованных атомов бора в $\delta$-допированном слое и поэтому сильно возрастает с увеличением ее средней кинетической энергии и степени экранирования этих потенциалов другими дырками. Эта степень (а в случае вырожденного двумерного газа дырок и эта энергия) резко падает при уменьшении двумерной концентрации дырок в $\delta$-допированном слое проводящего канала полевого транзистора при подаче на его затвор обедняющего напряжения. В результате уменьшение подвижности дырок с падением их двумерной концентрации при обеднении $\delta$-допированного слоя затворным напряжением может усилить эффект модуляции проводимости проводящего канала (пропорциональной произведению подвижности на двумерную концентрацию) и, следовательно, тока исток-сток.

Эффект уменьшения подвижности носителей заряда при снижении их двумерной концентрации обедняющим напряжением известен в физике обычных полупроводников, например для электронов в $\mathrm{Si}$-допированных квантовых ямах InGaAs в AlGaAs в проводящих каналах полевых транзисторов [16-18]. Однако при соответствующих вычислениях подвижности носителей заряда их рассеяние на ионизованных атомах допирующей примеси обычно рассматривается в борновском приближении, т.е. в рамках теории возмущений (см., например, [19]). Для алмаза, однако, это приближение несправедливо (см. далее разд. 3). Настоящая работа посвящена аналитическому рассмотрению и численному моделированию зависимости подвижности дырок в $\delta$-допированном проводящем канале полевого транзистора на алмазе от их двумерной концентрации, управляемой напряжением на затворе, без использования борновского приближения, т.е. теории возмущений. Ее целью является корректное (для параметров алмаза) предсказание начальной величины этой концентрации, при которой данная зависимость будет наиболее резкой и, следовательно, модуляция тока исток-сток напряжением на затворе наиболее эффективной. Для этого в следующем разделе найдена форма экранированного двумерным газом дырок кулоновского потенциала ионизованного атома бора в зависимости от его концентрации. В разд. 3 без использования борновского приближения (т.е. вне рамок теории возмущений) вычислено транспортное сечение рассеяния дырок на таком потенциале и определена их подвижность как функция их двумерной концентрации при обеднении $\delta$-допированного слоя напряжением на затворе. В Заключение даны основанные на полученных результатах рекомендации для повышения эффективности модуляции тока исток-сток напряжением на затворе в полевых транзисторах на алмазе с $\delta$-допированными проводящими каналами.

\section{2. Экранированный двумерным дырочным газом кулоновский потенциал}

Пусть $\delta$-допированный слой параллелен плоскости $x y$ и его центральная плоскость имеет координату $z=0$. Уравнение Пуассона для электростатического потенциала $\varphi$ имеет вид

$$
\varepsilon \Delta \varphi=-4 \pi \rho,
$$

где $\varepsilon \approx 5.7$ - электростатическая диэлектрическая проницаемость алмаза, $\rho=\rho_{0}+\tilde{\rho}-$ плотность заряда, $\rho_{0}=e \delta(x) \delta(y) \delta\left(z-z_{0}\right)$ - плотность внешнего заряда, создаваемого ионизованным атомом бора с зарядом $e<0$ (равным заряду электрона), расположенным в точке с координатами $\left(0,0, z_{0}\right), \tilde{\rho}$ - экранирующая внешний заряд плотность заряда, создаваемая за счет отклонения распределения дырок от однородного. Для выражения $\tilde{\rho}$ через $\varphi$ представим их в виде двумерных интегралов Фурье $\varphi=\int_{-\infty}^{+\infty} \int_{-\infty}^{+\infty} \exp (i \mathbf{q r}) \varphi_{\mathbf{q}}(z) d^{2} q /(2 \pi)^{2}$ (и аналогично для $\tilde{\rho})$, где $\mathbf{r}-$ двумерный радиус-вектор, лежащий в плоскости $x y$. Тогда (1) примет вид

$$
\begin{gathered}
d^{2} \varphi_{\mathbf{q}} / d^{2} z-q^{2} \varphi_{\mathbf{q}}+4 \pi \tilde{\rho}_{\mathbf{q}} / \varepsilon=-4 \pi e \delta\left(z-z_{0}\right) / \varepsilon \\
\tilde{\rho}_{\mathbf{q}}=-q_{s}(q) \int_{-\infty}^{+\infty} \varphi_{\mathbf{q}}\left(z^{\prime}\right)\left|\psi\left(z^{\prime}\right)\right|^{2} d z^{\prime}|\psi(z)|^{2} \\
q_{s}(q)=e^{2} \int_{-\infty}^{+\infty} \int_{-\infty}^{+\infty} \frac{f_{\mathrm{FD}}(\mathbf{k}-\mathbf{q} / 2)-f_{\mathrm{FD}}(\mathbf{k}+\mathbf{q} / 2)}{\hbar^{2} \mathbf{k q} / m} \frac{2 d^{2} k}{(2 \pi)^{2}}
\end{gathered}
$$

где $q_{s}(q)$ - линдхардовская восприимчивость [20], имеющая в двумерном случае размерность волнового числа, $\psi(z)$ - волновая функция дырок, описывающая их распределение в ортогональном к плоскости $\delta$-допированного слоя направлении, $f_{\mathrm{FD}}(\mathbf{k})$ - равновесная функция распределения дырок Ферми-Дирака, $\mathbf{k}$ - двумерный квазиволновой вектор дырки, лежащий в плоскости $x y, m$ - эффективная масса дырки, $\hbar-$ постоянная Планка, $N_{s}=2 /(2 \pi)^{2} \int_{-\infty}^{+\infty} \int_{-\infty}^{+\infty} f_{\mathrm{FD}}(\mathbf{k}) d^{2} k-$ двумерная концентрация дырок ${ }^{1}$.

Вычисление функции $q_{s}(q)$ может быть сравнительно легко выполнено в двух предельных случаях, когда энергия Ферми для двумерного дырочного газа

\footnotetext{
1 Здесь для простоты считается, что в потенциальной яме, создаваемой $\delta$-допированным слоем, формируется только одна дырочная подзона. Однако детальный вывод линдхардовского выражения для $\tilde{\rho}_{\mathbf{q}}$ при учете нескольких подзон (основных и возбужденных подзон тяжелых, легких и спин-отщепленных дырок) показывает, что в зависимости от параметров $\delta$-допированного слоя $\tilde{\rho}_{\mathbf{q}}$ может увеличиться только в несколько раз по сравнению с (3). Это не приведет к качественному изменению полученных далее результатов.
} 
$E_{\mathrm{F}} \equiv 2 \pi \hbar^{2} N_{s} /(2 m)$ много меньше или больше тепловой энергии $k_{\mathrm{B}} T$, где $k_{\mathrm{B}}$ - постоянная Больцмана, $T-$ температура. В первом случае (невырожденный двумерный газ)

$$
q_{s}(q)=\frac{\sqrt{\pi} e^{2} N_{s}}{2 k_{\mathrm{B}} T} \frac{k_{\mathrm{th}}}{q}\left(-q^{2} / k_{\mathrm{th}}^{2}\right) \operatorname{erfi}\left(q / k_{\mathrm{th}}\right),
$$

где $k_{\mathrm{th}}=\sqrt{8 m k_{\mathrm{B}} T / \hbar^{2}}-$ тепловое волновое число дырок, $\operatorname{erfi}(\xi) \equiv \operatorname{erfi}(\xi) / i, \operatorname{erf}-$ интеграл вероятностей [21]. Во втором случае (вырожденный двумерный газ)

$$
q_{s}(q)=\frac{e^{2} m}{\pi \hbar^{2}} \begin{cases}1, & q<2 k_{\mathrm{F}}, \\ 1-\sqrt{1-4 k_{\mathrm{F}}^{2} / q^{2}}, & q>2 k_{\mathrm{F}}\end{cases}
$$

где $k_{\mathrm{F}} \equiv \sqrt{2 m E_{\mathrm{F}} / \hbar^{2}}-$ волновое число Ферми. В обоих случаях при малых $q\left(q \ll k_{\text {th }}\right.$ в первом случае и $q<2 k_{\mathrm{F}}$ во втором), когда для вычисления $\tilde{\rho}_{\mathbf{q}}$ можно использовать квазиклассическое приближение, из (4) и (5) следует, что $q_{s}$ не зависит от $q$ и дается формулами $q_{s}(0)=e^{2} N_{s} /\left(k_{\mathrm{B}} T\right)$ в первом случае и $q_{s}(0)=e^{2} m /\left(\pi \hbar^{2}\right)$ во втором. Обе эти формулы есть соответствующие предельные виды общей формулы $q_{s}(0)=e^{2} \partial N_{s} / \partial \xi$, где $\xi-$ химический потенциал двумерного дырочного газа (во втором случае совпадающий с $\left.E_{\mathrm{F}}\right)$. При больших $q\left(q \gg k_{\text {th }}\right.$ в первом случае и $q \gg 2 k_{\mathrm{F}}$ во втором) $q_{s}(q)$ уменышается с увеличением $q$ пропорционально $1 / q^{2}$.

Для простоты будем считать, что $|w(z)|^{2}=1 / \Delta z$ $=$ const при $-\Delta z / 2<z<\Delta z / 2$ и 0 при остальных $z$. Тогда легко найти решение (2), удовлетворяющее очевидным условиям $\varphi_{\mathbf{q}} \rightarrow 0$ при $|z| \rightarrow \infty$, непрерывности $\varphi_{\mathbf{q}}$ при $z=z_{0}$, а также $d \varphi_{\mathbf{q}} /\left.d z\right|_{z_{0}+0}-d \varphi_{\mathbf{q}} /\left.d z\right|_{z_{0}-0}=4 \pi e / \varepsilon$. Из него следует, что

$$
\begin{aligned}
& \int_{-\Delta z / 2}^{+\Delta z / 2} \varphi_{\mathbf{q}}\left(z^{\prime}\right)\left|\psi\left(z^{\prime}\right)\right|^{2} d z^{\prime}=\frac{4 \pi e q \Delta z}{\varepsilon} \\
& \quad \times \frac{1-\exp (-q \Delta z / 2) \cosh \left(q z_{0}\right)}{2 q_{s}(q)[\exp (-q \Delta z)-1]+2 q_{s}(q) q \Delta z+q^{3}(\Delta z)^{2}} .
\end{aligned}
$$

Используя (6), можно вычислить зависимость от $\mathbf{r}$ электростатического потенциала, усредненного по поперечному к $\delta$-допированному слою распределению дырок:

$$
\begin{aligned}
u(r) & \equiv \int_{-\infty}^{+\infty} \int_{-\infty}^{+\infty} \exp (i \mathbf{q r}) \int_{-\Delta z / 2}^{+\Delta z / 2} \varphi_{\mathbf{q}}\left(z^{\prime}\right)\left|\psi\left(z^{\prime}\right)\right|^{2} d z^{\prime} \frac{d^{2} q}{(2 \pi)^{2}} \\
& =\int_{0}^{+\infty} J_{0}(q r) \int_{-\Delta z / 2}^{+\Delta z / 2} \varphi_{\mathbf{q}}\left(z^{\prime}\right)\left|\psi\left(z^{\prime}\right)\right|^{2} d z^{\prime} \frac{q d q}{2 \pi}
\end{aligned}
$$

где интегрирование по углу $\theta$ между $\mathbf{q}$ и $\mathbf{r}$ выполнено с помощью формулы $\int_{0}^{2 \pi} \exp (i q r \cos \theta) d \theta=2 \pi J_{0}(q r)$, в которой $J_{0}-$ функция Бесселя нулевого порядка [21].

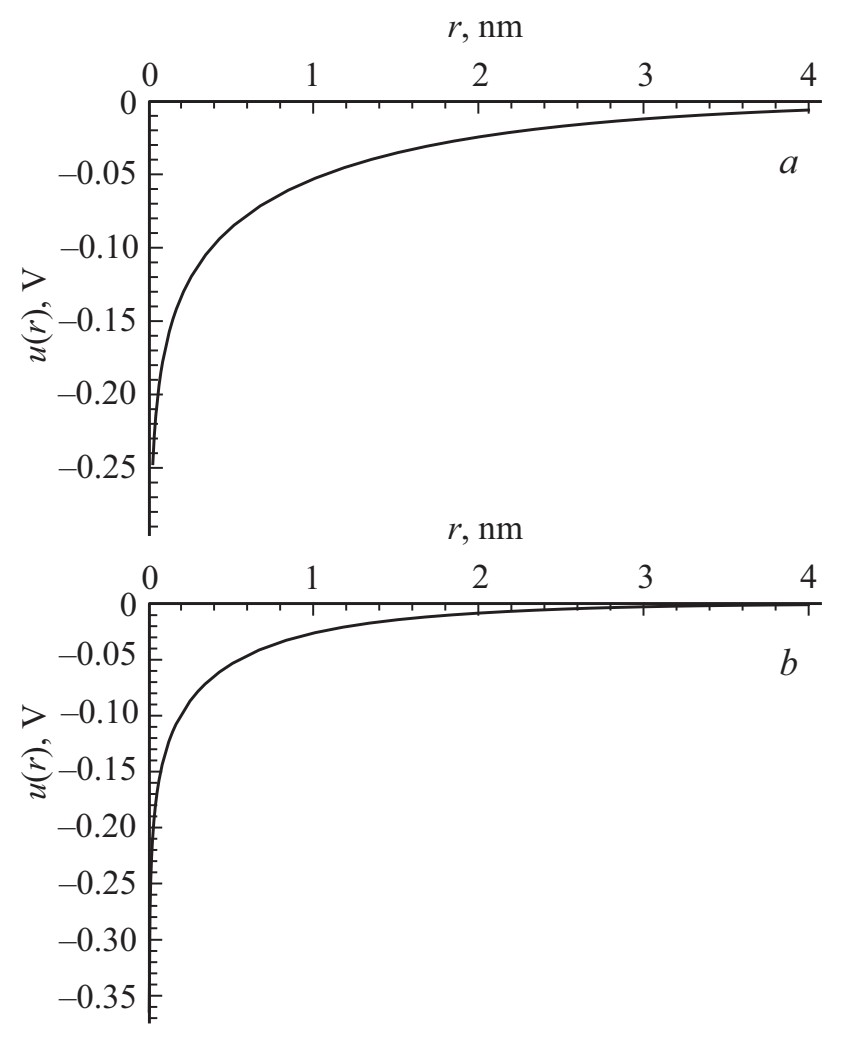

Рис. 1. Экранированный кулоновский электростатический потенциал $u(r)$ ионизованного атома бора, расположенного в центральной плоскости $\delta$-допированного слоя $\left(z_{0}=0\right)$. Панель $a$ отвечает $N_{s}=1.6 \cdot 10^{12} \mathrm{~cm}^{-2}$, панель $b-$ $N_{s}=2.5 \cdot 10^{13} \mathrm{~cm}^{-2}$.

При комнатной температуре $T=300 \mathrm{~K}$ и эффективной массе дырок $m=0.588 m_{0}$ (принимаемой для нижеследующих оценок равной эффективной массе тяжелых дырок [22], поскольку эффективные массы легких и спин-отщепленных дырок в алмазе близки к ней), где $m_{0}$ - масса свободного электрона, равенство $E_{\mathrm{F}}=k_{\mathrm{B}} T$ отвечает $N_{s 0}=6.35 \cdot 10^{12} \mathrm{~cm}^{-2}$. Для $N_{s}=0.25 N_{s 0}=1.6 \cdot 10^{12} \mathrm{~cm}^{-2}$, когда $E_{\mathrm{F}} \ll k_{\mathrm{B}} T$, т. е. peализуется случай невырожденного двумерного газа и $q_{s}(q)$ дается (4), зависимость $u(r)$ показана на рис. $1, a$. Для $N_{s}=4 N_{s 0}=2.5 \cdot 10^{13} \mathrm{~cm}^{-2}$, когда $E_{\mathrm{F}} \gg k_{\mathrm{B}} T$, т. е. имеет место противоположный случай вырожденного двумерного газа дырок и $q_{s}(q)$ дается (5), зависимость $u(r)$ приведена на рис. $1, b$. В обоих случаях положено $\Delta z=10$ нм.

Из рис. 1 видно, что с ростом двумерной концентрации дырок $u(r)$ спадает с увеличением $r$ быстрее, т.е. экранирование становится более эффективным. В обоих случаях при малых $r$ потенциал стремится к $-\infty$ пропорционально $\ln (r)$ (как легко установить, используя при интегрировании по $q$ в (7) асимптотику (6) при больших $q$ и предельное значение $J_{0}(g r)$ при малых $q r$ [21]), а при больших $r-$ к нулю пропорционально $1 / r^{2}$ (как легко убедиться, беря для $J_{0}(q r)$ ее асимптотику при больших $q r$ [21], представляя 
входящий в нее косинус в виде суммы двух экспонент и изменяя для каждой экспоненты контур интегрирования по $q$ в (7) с положительной вещественной полуоси на положительную или отрицательную мнимые полуоси так, чтобы эта экспонента затухала при удалении от начала координат). Отличие поведения $u(r)$ при малых $r$ от чисто кулоновского есть следствие выполненного в (7) усреднения по поперечной к $\delta$-допированному слою координате. Не экспоненциальное, а степенное спадание $u(r)$ при больших $r$ характерно для линдхардовской теории экранировки [20] и двумерных систем [23]. Конкретные критерии малости или величины расстояния зависят от соотношения имеющих размерность длины параметров $\Delta z, 1 / k_{\mathrm{th}}, 1 / q_{s}(0)$ и $\Delta z, 1 / k_{\mathrm{F}}, 1 / q_{s}(0)$ в случаях невырожденного и вырожденного двумерного газа дырок соответственно.

\section{3. Рассеяние дырок на экранированном кулоновском потенциале в двух измерениях}

Численные оценки показывают, что в алмазе для описания рассеяния дырок на найденном выше экранированном кулоновском электростатическом потенциале $u(r)$ (как в невырожденном, так и в вырожденном случаях) борновское приближение (т. е. теория возмущений) [24] неприменимо. Поэтому для рассмотрения рассеяния дырок на $u(r)$ используем точное двумерное стационарное уравнение Шредингера для не зависящей от времени волновой функции дырки $\phi(r, \theta)$ (где $\theta-$ угол между $\mathbf{r}$ и осью $x$ ), описывающей ее квазисвободное движение в плоскости $\delta$-допированного слоя $x y^{2}$ :

$$
-\frac{\hbar^{2}}{2 m}\left[\frac{1}{r} \frac{\partial}{\partial r}\left(r \frac{\partial \phi}{\partial r}\right)+\frac{1}{r^{2}} \frac{\partial^{2} \phi}{\partial \theta^{2}}\right]-e u(r) \phi=E \phi .
$$

Здесь $E-$ положительная энергия квазисвободного двумерного движения дырки, $-e>0-$ ее заряд. Его решение для двумерного движения с сохраняющейся (вследствие независимости $u(r)$ от $\theta$ ) проекцией момента импульса на ортогональную $\delta$-допированному слою ось $z$, равной (в единицах $\hbar) l=0, \pm 1, \pm 2, \ldots$, может быть представлено в виде $\phi_{l}=R_{l}(r) \exp (i l \theta)$, где $R_{l}$ удовлетворяет уравнению

$$
-\frac{\hbar^{2}}{2 m}\left[\frac{1}{r} \frac{\partial}{\partial r}\left(r \frac{\partial R_{l}}{\partial r}\right)-\frac{l^{2} R_{l}}{r^{2}}\right]-e u(r) R_{l}=E R_{l} .
$$

\footnotetext{
2 Для простоты считаем, что процесс рассеяния дырки не приводит к изменению номера и типа подзоны, в которой она первоначально находилась, и, вследствие близости эффективных масс тяжелых, легких и спин-отщепленных дырок в алмазе, для нижеследующих оценок полагаем, что эффективные массы дырок во всех подзонах одинаковы и равны эффективной массе тяжелой дырки. Учет межподзонного рассеяния может привести к уменышению полученной далее подвижности дырок, однако не скажется существенно на относительном ее изменении при вариации двумерной концентрации дырок в $\delta$-допированном слое.
}

Для больших $r$, при которых $|e u| \ll E$, справедлива асимптотическая формула $R_{l} \propto-J_{|l|}(k r)+\operatorname{tg}\left(\delta_{l}\right) Y_{|l|}(k r)$, где $k=\sqrt{2 m E} / \hbar-$ двумерное квазиволновое число, $J_{|l|}$ и $Y_{|l|}$ - функции Бесселя порядка $|l|$ первого и второго рода соответственно [21] (каждая из которых является решением (9) при больших $r), \delta_{l}=\delta_{-l}$ - зависящие от энергии и $l$ фазовые сдвиги [24], обусловленные наличием потенциала $u$ при малых $r\left(\delta_{l}=0\right.$ при $\left.u(r)=0\right)$. Последние находятся численно с помощью следующей процедуры.

Для малых $r$, при которых $|e u| \gg E$, в (9) можно пренебречь членом $E R_{l}$ по сравнению с $-e u(r) R_{l}$. Поскольку $r u(r) \rightarrow 0$ при $r \rightarrow 0$ (как это следует из результатов предыдущего раздела), то при $l=0$ для конечности $d^{2} R_{0} / d r^{2}$ при $r=0$ нужно, чтобы $d R_{0} / d r \rightarrow 0$ при $r \rightarrow 0$. Если же $l \neq 0$, то, вследствие $r u(r) \rightarrow 0$ при $r \rightarrow 0$, в (9) можно пренебречь членом $-e u(r) R$ по сравнению с $\hbar^{2} l^{2} R_{l} /\left(2 m r^{2}\right)$. Тогда решение (9) легко находится и имеет вид $R_{l}=a r^{|l|}+b r^{-|l|}$, где $a$ и $b-$ произвольные константы. Для конечности $R_{l}$ при $r \rightarrow 0$ необходимо, чтобы $b=0$, т. е. $\frac{r}{R_{l}} \frac{d R_{l}}{d r}$, при $r \rightarrow 0$ должно стремиться к $|l|$. Таким образом, получаем следующие граничные условия для (9) при $r=0: d R_{0} / d r=0$ для $l=0$ и $\frac{r}{R_{l}} \frac{d R_{l}}{d r}=|l|$ для $l \neq 0$. Затем, используя для $R_{0}$ и $R_{l}$ приведенные выше асимптотические выражения при некотором большом $r$, вычислим фазовые сдвиги $\delta_{0}$ и $\delta_{1}$ из условия, чтобы численное решение задачи Коши для (9) с начальными значениями функции и ее производной при $r$, полученными из этих асимптотических выражений, удовлетворяло при $r=0$ данным граничным условиям.

Используя найденные таким образом фазовые сдвиги, можно вычислить транспортное сечение рассеяния $\sigma_{\mathrm{tr}}$ (которое в рассматриваемом двумерном случае имеет размерность длины) [24]:

$$
\begin{aligned}
& \sigma_{\mathrm{tr}}=\int_{0}^{2 \pi}\left|\frac{1}{i \sqrt{2 \pi k}} \sum_{l=-\infty}^{+\infty}\left[\exp \left(2 i \delta_{l}\right)-1\right] \exp (i l \theta)\right|^{2} \\
& \times(1-\cos \theta) d \theta=\frac{1}{k}\left(4 \sum_{l=-\infty}^{+\infty} \sin ^{2}\left(\delta_{l}\right)-2 \sum_{l=0}^{+\infty}\left\{\left[1-\cos \left(2 \delta_{l}\right)\right]\right.\right. \\
& \left.\left.\times\left[1-\cos \left(2 \delta_{l+1}\right)\right]+\sin \left(2 \delta_{l}\right) \sin \left(2 \delta_{l+1}\right)\right\}\right) .
\end{aligned}
$$

Средняя скорость дырок $\overline{\mathbf{v}}(t)$ вычисляется по их функции распределения $f(\mathbf{k}, t)$ по формуле $\overline{\mathbf{v}}(t)=\frac{2}{(2 \pi)^{2} N_{s}} \int_{-\infty}^{+\infty} \int_{-\infty}^{+\infty} \frac{\hbar \mathbf{k}}{m} f(\mathbf{k}, t) d^{2} k$. Для неравновесной однородной в пространстве функции распределения в отсутствие внешних полей производная $d \overline{\mathbf{v}}(t) / d t$ определяется стандартным интегралом столкновений. В результате для рассматриваемых здесь упругих столкновений (считая для простоты, что все ионизованные атомы бора лежат в центральной плоскости $\delta$-допированного слоя, 
Сравнение вычисленных и экспериментальных значений подвижности дырок в $\delta$-допированных бором слоях алмаза

\begin{tabular}{c|c|c}
\hline \multirow{2}{*}{$N_{s}, \mathrm{~cm}^{-2}$} & \multicolumn{2}{|c}{$\mu, \mathrm{cm}^{2} /(\mathrm{B} \cdot \mathrm{c})$} \\
\cline { 2 - 3 } & Работа $[13]$ & Данный расчет \\
\hline $6.5 \cdot 10^{11}$ & 240 & 539.15 \\
$1.3 \cdot 10^{13}$ & 120 & 213.60
\end{tabular}

т. е. имеют координату $\left.z_{0}=0\right)$ имеем

$$
\frac{d \overline{\mathbf{v}}(t)}{d t}=-\frac{2 N_{0}}{(2 \pi)^{2} N_{s}} \int_{-\infty}^{+\infty} \int_{-\infty}^{+\infty} \sigma_{\mathrm{tr}} \frac{\hbar k}{m} \frac{\hbar \mathbf{k}}{m} f(\mathbf{k}, t) d^{2} k,
$$

где $N_{0}$ - двумерная концентрация ионизованных атомов бора в $\delta$-допированном слое.

Для нахождения времени релаксации $\tau$ малой скорости $\overline{\mathbf{v}}(t)$ к нулю рассмотрим модельную неравновесную функцию распределения $f(\mathbf{k}, t)=f_{\mathrm{FD}}[\mathbf{k}-m \overline{\mathbf{v}}(t) / \hbar]$, подставим ее в (11) и разложим по степеням $\overline{\mathbf{v}}(t)$ c точностью до линейных членов включительно. Это дает

$$
\begin{gathered}
\frac{d \overline{\mathbf{v}}(t)}{d t}=-\frac{\overline{\mathbf{v}}(t)}{\tau} \\
\frac{1}{\tau}=-\frac{2 N_{0}}{(2 \pi)^{2} N_{s}} \int_{-\infty}^{+\infty} \int_{-\infty}^{+\infty} \sigma_{\mathrm{tr}} k \frac{\hbar \mathbf{k}}{m} \frac{\partial f_{\mathrm{FD}}(\mathbf{k})}{\partial \mathbf{k}} d^{2} k .
\end{gathered}
$$

Эта общая формула упрощается в предельных случаях невырожденного и вырожденного двумерного дырочного газа, когда $f_{\mathrm{FD}}(\mathbf{k})$ имеет вид максвелловского или ступенчатого распределения соответственно. В первом случае имеем

$$
\frac{1}{\tau}=\frac{\sqrt{2} N_{0}}{\sqrt{m}\left(k_{\mathrm{B}} T\right)^{2}} \int_{0}^{+\infty} \sigma_{\mathrm{tr}} E^{3 / 2} \exp \left[-E /\left(k_{\mathrm{B}} T\right)\right] d E,
$$

во втором -

$$
\frac{1}{\tau}=N_{0} \sigma_{\mathrm{tr}} \hbar k_{\mathrm{F}} / m
$$

где $\sigma_{\mathrm{tr}}$ берется при $E=E_{\mathrm{F}}$. Для вычисления входящего в $(13 \mathrm{a})$ и $(13 \mathrm{~b}) \sigma_{\mathrm{tr}}$ фазовые сдвиги $\delta_{l}$ определяются для рассеивающего потенциала $u(r)$, найденного с использованием предельных формул (4) и (5) для $q_{s}(q)$ соответственно. По времени релаксации находим подвижность дырок $\mu=|e| \tau / m$.

Для тестирования описанного метода расчета подвижности дырок сравним получаемые с помощью него результаты с экспериментальными данными [13] (см. таблицу). Из таблицы видно, что рассчитанные подвижности по порядку величины совпадают с экспериментальными, однако оказываются в $\sim 2$ раза больше последних. По-видимому, это связано с тем, что для использованных в [13] образцов ширина $\Delta z$ распределения дырок в ортогональном плоскости $\delta$-допированного слоя направлении была значительно меньше, чем использованная в настоящих расчетах величина. Это приводит к более сильному, чем расчетное, рассеянию дырок на находящихся в $\delta$-допированном слое ионизованных атомах бора и, следовательно, меньшей их подвижности. Возможность такого объяснения поддерживается тем, что из измеренного в [13] для одного из образцов распределения дырок в ортогональном плоскости $\delta$-допированного слоя направлении можно заключить, что $\Delta z \approx 5$ нм, т.е. в $\sim 2$ раза меньше использованной в настоящих расчетах величины 10 нм. Однако в [13] данные по распределению дырок в ортогональном плоскости $\delta$-допированного слоя направлении приведены только для одного образца, поэтому корректное сравнение измеренных там подвижностей с результатами предлагаемой расчетной схемы требует дополнительных экспериментальных исследований.

На рис. 2 показана зависимость $\mu\left(N_{s}\right)$ для $N_{0}=2.5 \cdot 10^{13} \mathrm{~cm}^{-2} . \quad$ При $N_{s}<N_{s 0}$ для $1 / \tau$ использовалась формула (13a) (где для упрощения вычисления интеграла $\sigma_{\mathrm{tr}}$ было взято при $E$, равной тепловой энергии $\left.k_{\mathrm{B}} T\right)$, для $N_{s}>N_{s 0}-$ формула (13b). В обоих случаях суммирование по $l$ в $(10)$ выполнялось вплоть до достаточно больших их абсолютных значений, так что увеличение последних не меняло существенно $\sigma_{\mathrm{tr}}$.

Конечно, использование предельных формул для случаев невырожденного и вырожденного двумерного дырочного газа при вычислении $\mu$ для $N_{s}$ вблизи $N_{s 0}$, строго говоря, несправедливо. Причина использования такого приближения в том, что в этих предельных случаях верны сравнительно простые формулы (4) и (5) для функции $q_{s}(q)$ (которая определяет форму $u(r)$ и, следовательно, $\left.\delta_{l}\right)$, что делает возможным компьютерное вычисление $\delta_{l}$ за разумное время. Тем не менее, как видно из рис. 2, разница между найденными таким образом $\mu$ при $N_{s}=N_{s 0}$ составляет всего 25\%. Это

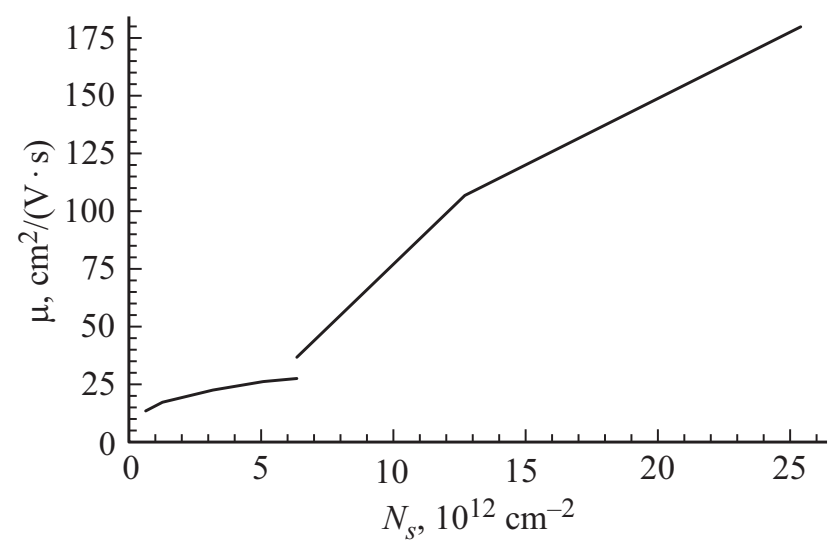

Рис. 2. Подвижность дырок как функция $N_{s}$. Небольшой разрыв кривой при $N_{s}=N_{s 0}=6.35 \cdot 10^{12} \mathrm{~cm}^{-2}$ объясняется использованием при вычислении $\mu$ предельных формул для невырожденного и вырожденного двумерного дырочного газа для меньших и больших $N_{s}$ соответственно. 
косвенно свидетельствует в пользу предположения, что использованное приближение дает качественно верную оценку $\mu\left(N_{s}\right)$ для $N_{s}$ вблизи $N_{s 0}$.

Как следует из рис. 2, подвижность дырок резко уменьшается при снижении $N_{s}$ в области больших значений последней: $\mu$ уменьшается в $\sim 5$ раз при снижении $N_{s}$ в 4 раза от $2.5 \cdot 10^{13}$ до $N_{s 0}=6.35 \cdot 10^{12} \mathrm{~cm}^{-2}$. Для меньших $N_{s}$ падение $\mu$ при снижении $N_{s}$ сохраняется, но становится значительно более плавным. Физическая причина такого поведения $\mu\left(N_{s}\right)$ заключается в происходящих при уменьшении двумерной концентрации дырок снижении степени экранировки рассеивающих кулоновских потенциалов ионизованных атомов бора и (в вырожденном случае) увеличении эффективности рассеяния дырок на них за счет уменьшения средней кинетической энергии последних.

Резкая начальная (считая от верхней границы значений $N_{s}$ на рис. 2) часть зависимости $\mu\left(N_{s}\right)$ может быть использована для увеличения степени модуляции тока исток-сток напряжением на затворе в алмазных полевых транзисторах с $\delta$-допированными проводящими каналами. Уменьшение $n_{s}$ в 4 раза приводит к снижению почти в $4 \cdot 5=20$ раз проводимости такого канала (пропорциональной $\mu N_{s}$ ) и, следовательно, тока исток-сток. Верхняя граница значений $N_{s}$ на рис. 2 выбрана из условия, чтобы газ дырок с такой двумерной концентрацией мог бы быть еще полностью вытеснен из $\delta$-допированного проводящего канала напряжением на затворе, отвечающим максимально возможному обедняющему электрическому полю порядка пробойного (которое в алмазе близко к $10 \mathrm{MB} / \mathrm{cm}[1]$ ).

\section{4. Заключение}

Таким образом показано, что подвижность дырок уменьшается при падении их двумерной концентрации в $\delta$-допированных бором слоях осажденного из газовой фазы алмаза (при условии неизменности двумерной концентрации рассеивателей - ионизованных атомов бора). При этом данное уменьшение тем резче, чем больше начальная двумерная концентрация дырок. Физическая причина этого эффекта заключается в происходящих при уменьшении двумерной концентрации дырок снижении степени экранировки рассеивающих кулоновских потенциалов ионизованных атомов бора и (в вырожденном случае) увеличении эффективности рассеяния дырок на них за счет уменьшения средней кинетической энергии последних.

Предсказанный эффект может быть использован для увеличения степени модуляции тока исток-сток напряжением на затворе в алмазных полевых транзисторах с $\delta$-допированными бором проводящими каналами. Из сказанного выше следует, что для этого необходимо создавать $\delta$-допированные слои с максимально допустимой (с точки зрения возможности ее существенного умень- шения затворным напряжением без пробоя алмаза) двумерной концентрацией дырок на уровне $3 \cdot 10^{13} \mathrm{~cm}^{-2}$.

\section{Финансирование работы}

Работа выполнена при поддержке правительства РФ (распоряжение № 220 от 9 апреля 2010 г., соглашение № 14.B25.31.0021).

\section{Конфликт интересов}

Автор заявляет об отсутствии конфликта интересов.

\section{Список литературы}

[1] Physics and Applications of CVD Diamond, ed. by S. Koizumi, C. Nebel, and M. Nesladek (Weinheim, Germany, Wiley-VCH Verlag GmbH \& Co. KGaA, 2008).

[2] H. Kato, K. Oyama, T. Makino, M. Ogura, D. Takeuchi, S. Yamasaki. Diamond Relat. Mater., 27-28, 19 (2012).

[3] T. Iwasaki, J. Yaita, H. Kato, T. Makino, M. Ogura, D. Takeuchi, H. Okushi, S. Yamasaki, M. Hatano. IEEE Electron Dev. Lett., 35, 241 (2014).

[4] H. Umezawa, T. Matsumoto, S.-I. Shikata. IEEE Electron Dev. Lett., 35, 1112 (2014).

[5] E.E. Schubert In: Semiconductors and Semimetals, 40 (N.Y., Academic Press Inc, 1994) Chap. 1.

[6] A. Fiori, T.N. Tran Thi, G. Chicot, F. Jomard, F. Omnès, E. Gheeraert, E. Bustarret. Diamond Relat. Mater., 24, 175 (2012).

[7] D. Araujo, M.P. Alegre, J.C. Pinero, A. Fiori, E. Bustarret, F. Jomard. Appl. Phys. Lett., 103, 042104 (2013).

[8] A. Fiori, F. Jomard, T. Teraji, S. Koizumi, J. Isoya, E. Gheeraert, E. Bustarret. Appl. Phys. Express, 6, 045801 (2013).

[9] A. Fiori, F. Jomard, T. Teraji, G. Chicot, E. Bustarret. Thin Sol. Films, 557, 222 (2014).

[10] P.N. Volpe, N. Tranchant, J.C. Arnault, S. Saada, F. Jomard, P. Bergonzo. Phys. Status Solidi Rapid Res. Lett., 6, 59 (2012).

[11] H. Shiomi, Y. Nishibayashi, N. Toda, S. Shikata. IEEE Electron Dev. Lett., 16, 36 (1995).

[12] A. Vescan, P. Gluche, W. Ebert, E. Kohn. IEEE Electron Dev. Lett., 18, 222 (1997).

[13] J.E. Butler, A. Vikharev, A. Gorbachev, M. Lobaev, A. Muchnikov, D. Radischev, V. Isaev, V. Chernov, S. Bogdanov, M. Drozdov, E. Demidov, E. Surovegina, V. Shashkin, A. Davidov, H. Tan, L. Meshi, A.C. Pakpour-Tabrizi, M.-L. Hicks, R.B. Jackman. Phys. Status Solidi Rapid Res. Lett., 11, 1600329 (2017).

[14] A. Aleksov, A. Vescan, M. Kunze, P. Gluche, W. Ebert, E. Kohn, A. Bergmaier, G. Dollinger. Diamond Relat. Mater., 8, 941 (1999).

[15] H. El-Hajj, A. Denisenko, A. Kaiser, R.S. Balmer, E. Kohn. Diamond Relat. Mater., 17, 1259 (2008).

[16] M.S. Shur, J.K. Abrokwah, R.R. Daniels, D.K. Arch. J. Appl. Phys., 61, 1643 (1987).

[17] K. Steiner, H. Mikami, K. Nishihori, N. Uchitomi, Jpn. J. Appl. Phys., 30, 29 (1991).

[18] M.H. Somerville, D.R. Greenberg, J.A. del Alamo. Appl. Phys. Lett., 64, 3276 (1994). 
[19] J.L. Thobel, L. Baudty, F. Dessenne, M. Charef, R. Fauquembergue. J. Appl. Phys., 73, 233 (1993).

[20] Н. Ашкрофт, Н. Мермин. Физика твердого тела, (М., Мир, 1979) т. 1, гл. 17. [N.W. Ashcroft, N.D. Mermin. Solid State Physics (N.Y., Holt, Rinehart and Winston, 1976) Chap. 17].

[21] У. Гаучи, Ф. Олвер. В кн.: Справочник по специальным бункциямб под ред. М. Абрамовица и И. Стиган (М., Наука, 1979) гл. 7,9. [W. Gautschi, F. Olver. In Handbook of Mathematical Functions, ed. by M. Abramowitz, I. Stegun (USA, National Bureau of Standards, Applied Mathematics Series 55, 1964) chs. 7,9].

[22] J. Pernot, P.N. Volpe, F. Omnès, P. Muret, V. Mortet, K. Haenen, T. Teraji. Phys. Rev. B, 81, 205203 (2010).

[23] T. Ando, A.B. Fowler, F. Stern. Rev. Mod. Phys., 54 (2), 437 (1982).

[24] Л.Д. Ландау, Е.М. Лифшиц. Квантовая механика. Нерелятивистская теория (М., Наука, 1989) гл. 17. [L.D. Landau, E.M. Lifshitz. Quantum Mechanics (Nonrelativistic Theory) (Oxford, UK, Butterworth-Heinemann, 2003) Chap. 17].

Редактор Г.А. Оганесян

\title{
Sharp drop of the mobility of holes with the decrease of their two-dimensional concentration by an external voltage in boron delta-doped diamond layers
}

\section{V.A. Kukushkin}

Institute of Applied Physics,

Russian Academy of Science, 603950 Nizhny Novgorod, Russia

Lobachevsky State University of Nizhny Novgorod, 603950 Nizhny Novgorod, Russia

\begin{abstract}
By analytical and numerical consideration it is shown that the mobility of holes in boron delta-doped (i.e. with thickness of order several lattice constants) layers in diamond drops with the decrease of their two-dimensional concentration in the process of the delta-doped layer depletion by an external voltage. This drop of the mobility is sharpest for maximal initial hole twodimensional concentrations of order $3 \cdot 10^{13} \mathrm{~cm}^{-2}$ (limited from above by the condition of the possibility of their substantial decrease without the electric breakdown of diamond) and is due to the significant mitigation of the screening degree of the ionized boron atom Coulomb potentials and the growth of the efficiency of the scattering of degenerate holes on them owing to the reduction of the kinetic energies of the latter. The corresponding calculations are carried out without the use of the Born approximation (i.e. the perturbation theory) because the conditions of its validity in boron delta-doped layers of diamond are not fulfilled. The predicted effect can be used to increase the source-to-drain current modulation by the gate voltage in diamond field-effect transistors with delta-doped conductive channels.
\end{abstract}

